\title{
Performant Container Support for HPC Applications
}

\author{
Yinzhi Wang \\ Texas Advanced Computing Center \\ Austin, Texas \\ iwang@tacc.utexas.edu
}

\author{
R. Todd Evans \\ Texas Advanced Computing Center \\ Austin, Texas \\ rtevans@tacc.utexas.edu
}

\author{
Lei Huang \\ Texas Advanced Computing Center \\ Austin, Texas \\ huang@tacc.utexas.edu
}

\begin{abstract}
The demand for the ability to easily customize, reproduce and migrate applications and workflows has been steadily increasing among the HPC community as software environments and applications grow in complexity. Lightweight containers that are suitable for HPC applications at scale are considered to be a viable approach to meet this demand. Previous studies have addressed the performance aspects of most existing containers using microbenchmarks and revealed the performance overheads of the best implementations to be small. However, the feasibility of providing containerized, real-world HPC applications on HPC systems, and the impact on overall application performance at scale, has not yet been explored. Here we present a basic feasibility and performance study using the Singularity container. We evaluate what is required to enable container images to utilize the high-speed fabric present on most HPC systems and explore their performance by comparing real-world applications run both within a container and in the absence of a container (natively). The results indicate lightweight Singularity images are a promising approach to the HPC communities demands for not only customizability, reproducibility and portability, but also performance.
\end{abstract}

\section{CCS CONCEPTS}

- General and reference $\rightarrow$ Performance; $\bullet$ Software and its engineering $\rightarrow$ Application specific development environments; Software performance; $\bullet$ Networks $\rightarrow$ Network performance analysis.

\section{KEYWORDS}

Singularity, HPC, container

\section{ACM Reference Format:}

Yinzhi Wang, R. Todd Evans, and Lei Huang. 2019. Performant Container Support for HPC Applications. In Practice and Experience in Advanced Research Computing (PEARC '19), fuly 28-August 1, 2019, Chicago, IL, USA. ACM, New York, NY, USA, ?? pages. https://doi.org/10.1145/3332186.3332226

Permission to make digital or hard copies of all or part of this work for personal or classroom use is granted without fee provided that copies are not made or distributed for profit or commercial advantage and that copies bear this notice and the full citation on the first page. Copyrights for components of this work owned by others than the author(s) must be honored. Abstracting with credit is permitted. To copy otherwise, or republish, to post on servers or to redistribute to lists, requires prior specific permission and/or a fee. Request permissions from permissions@acm.org.

PEARC '19, Fuly 28-August 1, 2019, Chicago, IL, USA

(c) 2019 Copyright held by the owner/author(s). Publication rights licensed to ACM. ACM ISBN 978-1-4503-7227-5/19/07 . .\$15.00

https://doi.org/10.1145/3332186.3332226

\section{INTRODUCTION}

Both the size and complexity of HPC clusters have been growing over the past decades to meet the increasing demand for computational power in the science community. Such growth is accompanied by the deployment of many-core processors as well as low-latency, high-bandwidth interconnect fabrics that require specialized hardware drivers to utilize. Effective utilization of the processors and fabric can extend the capability and capacity of scientific applications to execute workloads at extreme scales. The increasing complexity of the software environments these applications are developed in has led to a growing demand for more customizable HPC software environments to run them in. Although the necessity of interfacing with hardware drivers limits customizability, it is not obvious how significant these limitations are. This paper explores the trade-offs between customizability and performance when running HPC applications at scale.

The demand on customizability is effectively tackled by virtualization technologies, which have become prevalent due to their hardware independence, isolation, and security features. Combined with the concept of Grid Computing, virtualization technology established the new infrastructure known as cloud computing. The hypervisor-based virtualization solutions, such as Xen, VMware ES$\mathrm{X} / \mathrm{ESX}$, and KVM, are commonly implemented in the commercial cloud computing platforms. Their substantial performance overhead [?][?], however, has prevented the adoption of virtualization in the support of conventional HPC applications because they preclude processor specific optimizations and utilization of high speed fabrics due to the hypervisor layer.

Meanwhile, lightweight virtualization solutions such as containers that exclude the hypervisor layer have gained substantial traction in the HPC community. The container-based technology facilitating distribution and deployment of applications prevails in the research communities that emphasize the reproducibility of both scientific findings and computational environments. Docker [?] is among the most popular ones. However, due to the root privilege required to execute Docker containers and associated security concerns [? ], it cannot be easily adopted in most HPC environments. Singularity [?] is created for scientific application driven workloads to meet both the demands from users and administrators in the HPC environment. Singularity shares most of the benefits of the Docker container while mitigating the security concerns. Because of these characteristics the usage of Singularity, or similar container technologies, in the support of conventional HPC applications is likely to increase.

\subsection{Motivation}

Figure ?? compares the layers of software between an application running on a virtual machine (VM) and one running in a Singularity 


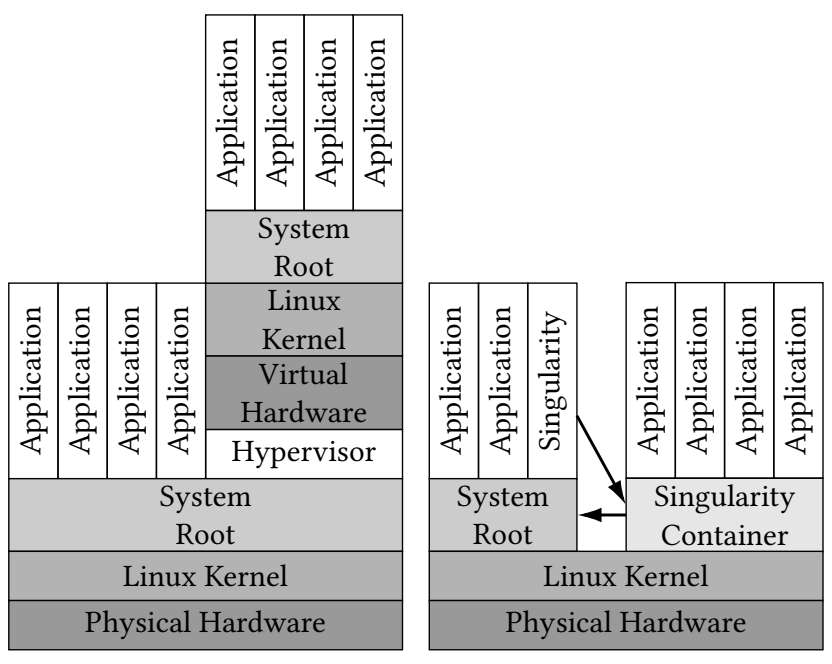

Figure 1: Architecture of hypervisor-based virtual environments (left), and Singularity as a container-based virtual environment (right). The Singularity daemon running along with other applications on the host launches the container. The applications running within the Singularity container can access system root through path binding or overlay.

container. While the VM obscures hardware resources from hosted applications with the interposition of the hypervisor, Singularity containers can expose the same hardware resources to container hosted applications as native applications. There are a number of studies that have analyzed the performance overhead of Singularity including its impact on disk I/O, Memory, and Network bandwidth in the HPC context [? ][? ][? ]. The microbenchmarks show negligible difference between running with or without the container (natively) and indicate that Singularity could be a great candidate to support HPC users requiring increased customizability, reproducibility and portability. For an HPC center, this involves more than just enabling Singularity on the clusters as a module for the users, but also releasing a selected number of basic container images that can be customized to fulfill user needs and still run seamlessly with all the native hardware to achieve high performance. We establish how effective a solution to combine customizability and performance in an HPC environment Singularity is by answering the following questions:

- What are the basic components required for containers to access the host's hardware?

- Are there any limitations on compatibility between different Linux distributions on the container and the host?

- How much performance overhead on real-world scientific applications is introduced by the container?

\subsection{Contribution}

To address these questions, we build two CentOS images, one of which has a compiler and MPI tools built into the container and the other which utilizes the host's compiler and MPI through Singularity's bind path mechanism. We also built a third container using the latest version of Ubuntu, with the host compiler and MPI exposed,
Table 1: Singularity Images Built

\begin{tabular}{lcc}
\hline OS Version & $\begin{array}{c}\text { Intel Compilers } \\
\text { and MPI }\end{array}$ & $\begin{array}{c}\text { Lmod } \\
\text { Module System }\end{array}$ \\
\hline CentOS 7.4.1708 & Built-in & Yes \\
CentOS 7.4.1708 & With bind path & Yes \\
Ubuntu 18.10 & With bind path & Yes \\
\hline
\end{tabular}

for comparison. Performance of the three images are evaluated running a set of commonly used scientific applications including WRF, MILC, NAMD, and GROMACS. The results show that these applications run on the images we built experience little to no measurable performance overhead compared to running the applications natively on the system. The results also show the presence of a small amount of constant overhead, due mostly to the startup of Singularity daemon. Through the binding of system directories, all these images can utilize all the pre-built tools and applications natively on the system and manage them with environment module systems such as Lmod[? ].

\section{IMPLEMENTATION}

\subsection{Resources}

All the experiments in this study run on the Stampede2 supercomputer at the Texas Advanced Computing Center. Stampede2 hosts 4,200 Knights Landing nodes and 1,736 Intel Xeon Skylake (SKX) nodes. We chose to use the SKX nodes to run all the tests to achieve better performance. Each of the SKX nodes has two Intel Xeon Platinum 8160 processors and a total of 192GB DDR4 memory. The interconnect of the system is a $100 \mathrm{~Gb} / \mathrm{sec}$ Intel Omni-Path (OPA) network.

\subsection{Singularity Images}

A total of three Singularity images are built (Table ??). The first one is based on CentOS Linux 7.4.1708 with the Intel compiler and MPI tools copied into the image during the build $\left(\mathrm{CentOS}_{1}\right)$. The second one is built with the same version of CentOS but with only the base GNU Compiler Collection (GCC) compiler included with CentOS in the container $\left(\mathrm{CentOS}_{2}\right)$. The third is built with Ubuntu 18.10 and has only Ubuntu's base GCC built-in. In addition to the basic tools and libraries, including proper versions of rdma and psm2 libraries for these Linux distributions, we also have Lmod installed to the same directory as the one on Stampede2 along with an initializing script so that the environment within the container is similar to the host's.

All three images are built in a CentOS Linux environment with root access on a personal computer. When running on Stampede2, in addition to the user writeable directories, /home1, /work, and /scratch, the staff maintained applications in /opt/apps are all mounted with the bind paths defined in Singularity. Exposing the host's /opt/intel directory then determines whether the host's Intel compiler and MPI are usable within the container.

The CentOS distribution that was running on Stampede 2 at the time of this study was identical to those in the CentOS containers, so the purpose of having the first two images is to minimize the discrepancy between the container and native system, thus all the 
pre-built modules on Stampede 2 are expected to run seamlessly when mounted within the container through bind paths. Having the Intel compilers and MPI built into one of the CentOS images is to further minimize potential issues due to the difference of system libraries. Then, the Ubuntu image represents the situation where most major system libraries are different inside the container. This corresponds to the cases where the user might demand some new features from the latest GNU C Library that is not available locally on an HPC system. With each of these three images, the users would have the freedom to install packages using the yum or apt-get command when building their own customized container images.

\subsection{Applications}

We picked four of the top 10 applications run on the Stampede2 supercomputer, WRF [? ], MILC [?], NAMD [? ], and GROMACS [?], to benchmark the performance of Singularity images. These application are from different scientific domains using different algorithms and should be representative of much of Stampede2's workload. Note that to have the comparison effective, we have four versions of each application built and compiled within the three container environments and the native Stampede 2 environment, and then run them all in the Stampede2 environment. All of the applications except for NAMD are compiled with the Intel Compiler using Intel MPI with the same optimization and vectorization options enabled to achieve the best possible performance. The NAMD version used here has known performance issues when compiled with newer versions of the Intel compiler and MPI. Given that NAMD is installed as a module on Stampede2, we instead compare its performance by loading the module within the container environment. The Intel Compiler and Intel MPI version is 18.0.2 if not specified otherwise in the following discussion.

The Weather Research and Forecasting (WRF) model is a numerical weather prediction application designed for atmospheric research and operational forecasting. It is based on a Eulerian solver using a third-order Runge-Kutta time-integration scheme coupled with a split, explicit second-order time integration scheme. The benchmark code MILC is used to study quantum chromodynamics, the theory of the strong interactions of subatomic physics. It's runtime is dominated by sparse matrix solver algorithms. NAMD is an application of classical molecular dynamics simulations that simulates the interaction between atoms. It integrates the forces on all atoms by the explicit, reversible, and symplectic Verlet algorithm to simulate the dynamic evolution of the system. GROMACS is a package to perform molecular dynamics using Newtonian equations of motion for systems with hundreds to millions of particles.

\subsection{Benchmark Descriptions}

- WRF Our WRF benchmarks uses the $2.5 \mathrm{KM}$ CONUS Benchmark dataset from http://www2.mmm.ucar.edu/wrf/WG2/bench/ Bench_V3_20081028.htm . In this dataset, the domain is $2.5 \mathrm{~km}$ in horizontal resolution on a 1500 by 1200 grid with 35 vertical levels with a time step of 15 seconds. We run the WRF benchmark on 1, 2, 4, 8, and 16 nodes each with 4 MPI tasks per node and and 12 OpenMP threads per task.
- MILC In this benchmark, the executable su3_rhmd_hisq evolves a 18x18x18x36 gauge configuration using a staggered-fermion rational hybrid Monte Carlo evolution code for 2 trajectories. The dataset is from https://portal.nersc.gov/project/m888/apex/ MILC_lattices/. The MILC benchmark is run on 3, 6, 9, and 18 nodes with 48 MPI tasks per node.

- NAMD This benchmark simulates the $1 \mathrm{M}$ atom Satellite Tobacco Mosaic Virus. It will be run on 1, 2, 4, 8, and 16 nodes with 4 MPI tasks per node, which is a more efficient configuration with multithreading enabled. The version of NAMD used is 2017_12_05 that is available as a module on Stampede2. It is built with Intel Compiler and Intel MPI version 16.0.3.

- GROMACS In this benchmark, pure water solutions were simulated using GROMACS version 2018.3. The simulated systems consist a total of 1.536 Million atoms. The initial coordinates and simulation parameters were obtained from GROMACS website at, ftp://ftp.gromacs.org/pub/benchmarkswater_GMX50_bare.tar. $\mathrm{gz}$. All simulations were performed in the isothermal isobaric (NpT) ensemble at $300 \mathrm{~K}$ and $1 \mathrm{~atm}$. Scaling behavior will be determined using 1, 2, 4, 8, and 16 nodes with 48 MPI tasks per node.

All the benchmarks run on the aforementioned four different environments for comparison. Each individual test is ran 3 times to take the average runtime.

\section{RESULTS AND DISCUSSION}

\subsection{Container Usage in an HPC Environment}

The two CentOS images build and run the applications flawlessly right out of the box. This is expected as both images have minimal differences from the native system. Building the Intel stack into the container image turns out to be unnecessary and redundant when a compiler on the host is available. This greatly improves the practicality of providing Intel-enabled images as products to users, because the Intel package itself is a multi-gigabyte package, and including it would significantly blow up the size and hence diskspace requirements and upload/download times for the container.

Due to the difference in the shared system libraries and headers, the mounted Intel compilers and MPI refuse to execute within the Ubuntu container. The compiler issue is specifically caused by the differences in the systems' math. $h$ headers between the two Linux distributions. We fixed it by substituting in Intel's math. $h$ header for the system version's within the Ubuntu container. This is achieved through defining __PURE_INTEL_C99_HEADERS__ when compiling. Intel MPI runtime issue only occurs when the incompatible GNU C Library is loaded. We fixed it by applying a customized patch with LD_PRELOAD to replace the original strtok_r() function implemented in Ubuntu 18.10 with our modified implementation shown as following:

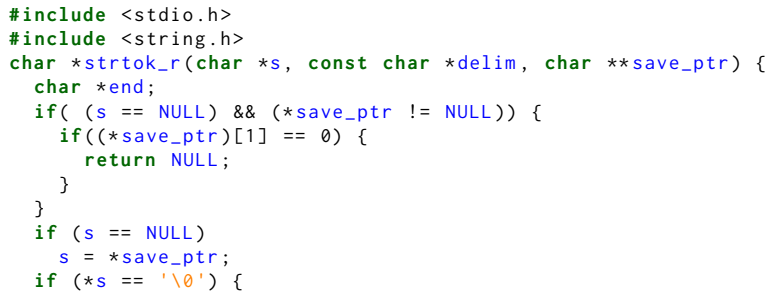




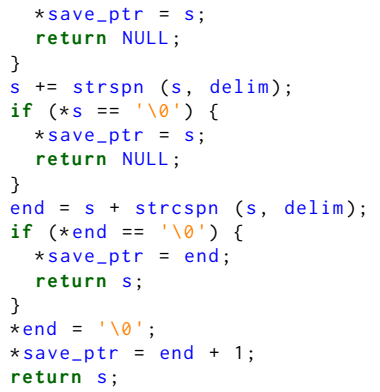

After the fixes, we were able to achieve the same workflow as in the CentOS images for all the benchmarks without any issues.

The GROMACS we built uses new features of $\mathrm{C}++11$ that revealed additional incompatibility between the Intel Compiler 18.0.2 and Ubuntu's native headers - specifically those provided in its base version 8.2.0 GCC. We fixed it by compiling the code with Intel Compiler 19.0.3 on the Ubuntu image. Due to noticeable performance improvement using the newer version of compiler, we also compiled GROMACS with the same version for the CentOS and native tests.

For the NAMD benchmarks, we used the executable from the pre-built namd/2017_12_05 module on Stampede2. This is another potential way of using the container for HPC users. Such that the users can use the built-in Lmod to manage and use all the modules available natively when inside the container. Due to the lack of older versions of Intel Compiler that the NAMD is built with, we excluded $\mathrm{CentOS}_{1}$ for this test. Both of the other container images have no issue running this native NAMD. Note that the patch for MPI is still necessary for Ubuntu.

\subsection{Application Benchmarks}

??????? show the results of all the benchmarks. In addition to the average runtime, the differences of maximum and minimum runtime measurements from the average are also given in the tables to reflect the uncertainty. Since we only run each tests three times, no statistical argument is implied from these numbers. Also, note that the runtime is recorded within the application, thus excludes the startup overhead of the container as well as the I/O time.

The runtime of all the WRF tests fluctuate quite a bit (up to $\sim 8 \%$ ) between runs (Table ??). This is especially obvious when the total runtime is small at 16 nodes. The overall difference between different system environments, however, is at the same level of fluctuation, and therefore insignificant.

The runtime of the MILC benchmark shows the same trend (Table ??). While the fluctuation is a little less than that of WRF, it still varies between runs (at $\sim 3 \%$ ). Again, no significant dependence on the environment is observed within the uncertainty across the tests.

The runtime of NAMD has a lot less variation compared to that of WRF and MILC (Table ??). Note that the CentOS $_{1}$ result is missing due to the required use of the Stampede2 NAMD module for this benchmark. There is also no significant performance sensitivity to environments for this NAMD benchmark.

GROMACS's result is similar to NAMD, where the variation is very small (Table ??). CentOS 2 's runtime at 2 nodes is noticeably
Table 2: WRF Benchmark Results (in seconds)

\begin{tabular}{|c|c|c|c|c|}
\hline Nodes & Native & CentOS $_{1}$ & CentOS $_{2}$ & Ubuntu \\
\hline 1 & $1252.6_{-66.0}^{+88 \cdot 4}$ & $\begin{array}{r}+9 \cdot 0 \\
1183.1+12 \cdot 8\end{array}$ & $1180.4_{-14 \cdot 0}^{+7 \cdot 8}$ & $\begin{aligned} 1172.1 & +5 \cdot 9 \\
& -3 \cdot 2\end{aligned}$ \\
\hline 2 & $\begin{array}{r}661.9^{+28 \cdot 0}-26 \cdot 1\end{array}$ & $676.7_{-29 \cdot 3}^{+25 \cdot 9}$ & $\begin{array}{r}641.7_{-21 \cdot 8}^{+27 \cdot 8} \\
-21 \cdot 1\end{array}$ & $653.6_{-35 \cdot 3}^{+60 \cdot 5}$ \\
\hline 4 & $370.5 \begin{array}{l}+3 \cdot 7 \\
-2 \cdot 6\end{array}$ & $\begin{array}{r}357.3+16 \cdot 3 \\
-11 \cdot 5\end{array}$ & $\begin{array}{r}+9 \cdot 4 \\
361.5 \\
-17 \cdot 7\end{array}$ & $\begin{array}{ll}354.3 & +4 \cdot 9 \\
-5 \cdot 9\end{array}$ \\
\hline 8 & $205.6 \begin{array}{r}+8 \cdot 5 \\
-13 \cdot 5\end{array}$ & $212.7 \begin{array}{l}+2 \cdot 7 \\
-4 \cdot 3\end{array}$ & $207.4 \begin{array}{l}+6 \cdot 4 \\
-8 \cdot 0\end{array}$ & $\begin{array}{r}208.7 \begin{array}{r}+7 \cdot 7 \\
-11 \cdot 2\end{array}\end{array}$ \\
\hline 16 & $\begin{array}{rr}+1 \cdot 3 \\
-1 \cdot 6 \\
\end{array}$ & $\begin{array}{r}+2 \cdot 7 \\
-1 \cdot 6 \\
\end{array}$ & $\begin{array}{r}140.6 \begin{array}{r}+12 \cdot 7 \\
-6 \cdot 4\end{array} \\
\end{array}$ & $133.6 \begin{array}{r}+0 \cdot 4 \\
-0 \cdot 8 \\
\end{array}$ \\
\hline
\end{tabular}

Table 3: MILC Benchmark Results (in seconds)

\begin{tabular}{|c|c|c|c|c|}
\hline Nodes & Native & CentOS $_{1}$ & CentOS $_{2}$ & Ubuntu \\
\hline 3 & $1106.2 \begin{array}{l}+39 \cdot 1 \\
-21 \cdot 7\end{array}$ & $1113.0 \begin{array}{l}+30 \cdot 4 \\
-21 \cdot 0\end{array}$ & $1086.0 \begin{array}{l}+23 \cdot 3 \\
-14 \cdot 1\end{array}$ & $1109.3_{-12 \cdot 1}^{+12 \cdot 3}$ \\
\hline 6 & $\begin{array}{ll}462.0 & +2 \cdot 0 \\
& -2 \cdot 0\end{array}$ & $467.6 \begin{array}{l}+0 \cdot 4 \\
-0 \cdot 6\end{array}$ & $461 .{ }^{+}+4 \cdot 0$ & $479.6_{-10 \cdot 8}^{+16 \cdot 0}$ \\
\hline 9 & $\begin{array}{ll} & +2 \cdot 8 \\
& \\
-1 \cdot 5\end{array}$ & $\begin{array}{ll} & +3 \cdot 2 \\
& \\
& -3 \cdot 7\end{array}$ & $381.9 \begin{array}{l}+4 \cdot 6 \\
-7 \cdot 0\end{array}$ & $\begin{array}{ll}381.2 & +6 \cdot 1 \\
-6 \cdot 8\end{array}$ \\
\hline 18 & $\begin{array}{ll}244.1 & +2 \cdot 0 \\
-1 \cdot 2\end{array}$ & $\begin{array}{ll}250.0 & +2 \cdot 1 \\
-3 \cdot 5\end{array}$ & $247.5 \begin{array}{l}+4 \cdot 3 \\
-2 \cdot 6\end{array}$ & $240.9 \begin{array}{l}+2 \cdot 1 \\
-1 \cdot 9\end{array}$ \\
\hline
\end{tabular}

Table 4: NAMD Benchmark Results (in seconds)

\begin{tabular}{|c|c|c|c|c|}
\hline Nodes & Native & CentOS $_{1}$ & $\mathrm{CentOS}_{2}$ & Ubuntu \\
\hline 1 & $231.8_{-1 \cdot 0}^{+0 \cdot 5}$ & - & $232 .{ }^{+0 \cdot 2}-0.3$ & $229.3_{-0 \cdot 7}^{+1 \cdot 1}$ \\
\hline 2 & $124.4_{-0.9}^{+0 \cdot 6}$ & - & $125.2_{-0.5}^{+0 \cdot 7}$ & $124.6_{-0.3}^{+0 \cdot 5}$ \\
\hline 4 & $\begin{array}{r}70.8^{+0 \cdot 5} \\
-0 \cdot 3\end{array}$ & - & 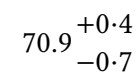 & $70.7_{-0.1}^{+0 \cdot 2}$ \\
\hline 8 & $\begin{array}{r}42.9^{+0 \cdot 2} \\
-0.3\end{array}$ & - & 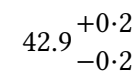 & $43.2_{-0 \cdot 0}^{+0 \cdot 1}$ \\
\hline 16 & $29 .{ }^{+0 \cdot 1}+0 \cdot 1$ & - & $29.6 \begin{array}{r}+0 \cdot 0 \\
-0.0\end{array}$ & $29.8 \begin{array}{l}+0 \cdot 0 \\
-0 \cdot 0\end{array}$ \\
\hline
\end{tabular}

slower due to one outlier among the three runs that go into this average number, thus it has no apparent relation to the environments that we are testing.

The runtime from all the benchmarks tested indicates the overhead due to containerization has almost no effect on the performance of the applications. In fact, occasionally the runs within a container outperform the native runs. This observation conforms with the microbenchmarks presented by others (e.g. [? ]), where the studied Singularity containers also used hardware resources directly. Our results indicate that all three Singularity images, with minimal and straightforward modifications, can efficiently execute the applications while providing the customizability, reproducibility and portability of a container. 
Table 5: GROMACS Benchmark Results (in seconds)

\begin{tabular}{|c|c|c|c|c|}
\hline Nodes & Native & CentOS $_{1}$ & $\mathrm{CentOS}_{2}$ & Ubuntu \\
\hline 1 & $233 .{ }_{-1 \cdot 3}^{+1 \cdot 0}$ & $234.0 \begin{array}{c}+0 \cdot 4 \\
-0.8\end{array}$ & $\begin{array}{r}234.9+2 \cdot 7 \\
-1 \cdot 8\end{array}$ & $234.5_{-0 \cdot 3}^{+0 \cdot 3}$ \\
\hline 2 & $117.9_{-0.5}^{+0.4}$ & $117.6_{-0.4}^{+0 \cdot 2}$ & $121.2_{-4 \cdot 2}^{+8 \cdot 1}$ & $115.2_{-0 \cdot 7}^{+0 \cdot 5}$ \\
\hline 4 & $61.4_{-0 \cdot 4}^{+0 \cdot 3}$ & $61.5_{-0.5}^{+0 \cdot 4}$ & $61.8_{-1 \cdot 0}^{+1 \cdot 9}$ & $\begin{array}{r}59.9+0 \cdot 4 \\
-0 \cdot 3\end{array}$ \\
\hline 8 & $\begin{array}{r}34.3+1 \cdot 1 \\
-0 \cdot 7\end{array}$ & $\begin{array}{r}34.9+1 \cdot 1 \\
-0 \cdot 7\end{array}$ & $33.6_{-0 \cdot 1}^{+0 \cdot 1}$ & $33.5_{-0 \cdot 1}^{+0 \cdot 2}$ \\
\hline 16 & $21.6_{-0.2}^{+0 \cdot 3}$ & $21.9^{+0 \cdot 2}$ & $21.4_{-0.2}^{+0 \cdot 2}$ & $21.1_{-0.4}^{+0.7}$ \\
\hline
\end{tabular}

\subsection{Overheads}

In addition to the runtime internally measured by each application, we also recorded the wall time for all the benchmarks. The difference between the two includes the time spent on starting up the application, reading and writing the data, setting up the problem, and so on. We group all of these contributions to overhead together in the following. Note that the wall time is measured outside of the container, thus also includes the time spent on starting up Singularity and loading the container image. Assuming the applications spent the same amount of time for other portions of the startup time excluding the overhead from container when run with the same problem size with the same node counts, we can estimate the overhead for each container image by subtracting the measured startup time of a benchmark for a container environment with that of the native environment. The container overhead, thus, includes not only the time spent on starting up Singularity and loading the container image but also any slowdown it caused for other phases such as I/O.

Figure ?? shows the startup time for WRF. For all four environments, the startup time scales down with increasing node counts. We attribute it to the excluded parallel I/O and problem setup phases in WRF's internal runtime measurement. Regardless, the startup time of CentOS ${ }_{1}$ is always much longer than the others, and it is more obvious at higher node counts.

The same startup time measurements from MILC benchmarks show no dependency on the node counts (Figure ??), which indicates negligible parallelism in the other part of the application. Again, we can see the startup time of CentOS $_{1}$ is much longer, and that of $\mathrm{CentOS}_{2}$ and Ubuntu is also slightly longer than Native runs.

The NAMD results in Figure ?? also show pretty consistent startup time across runs with node counts from 2 to 16 . When running with one node, the startup time of all three environments is $\sim 2$ s less than the rest. This is probably because all network communication related overheads are circumvented by NAMD when running in a shared memory environment.

The startup time of GROMACS benchmarks takes a relatively larger portion of the wall time due to the overall shorter runtime and longer startup time (Figure ??, Table ??). No dependency on the node counts is seen. The dependency on the environment, instead, is clearly shown, where the Ubuntu runs have the least startup time

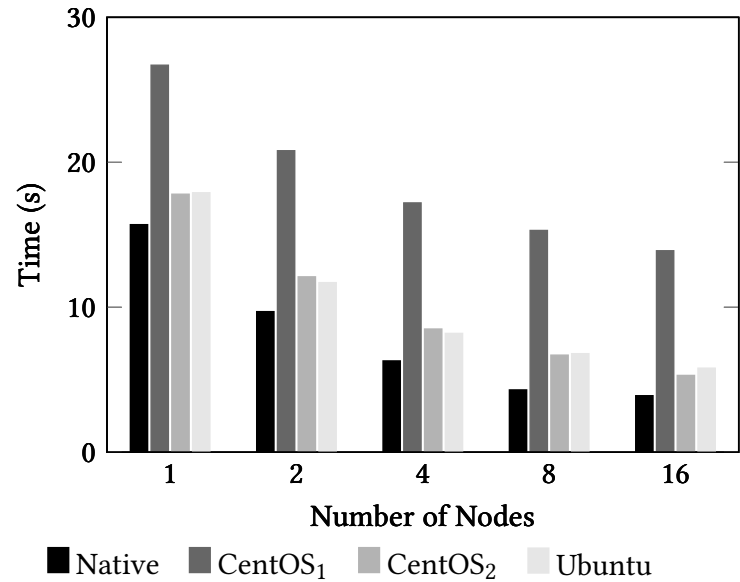

Figure 2: WRF startup time.

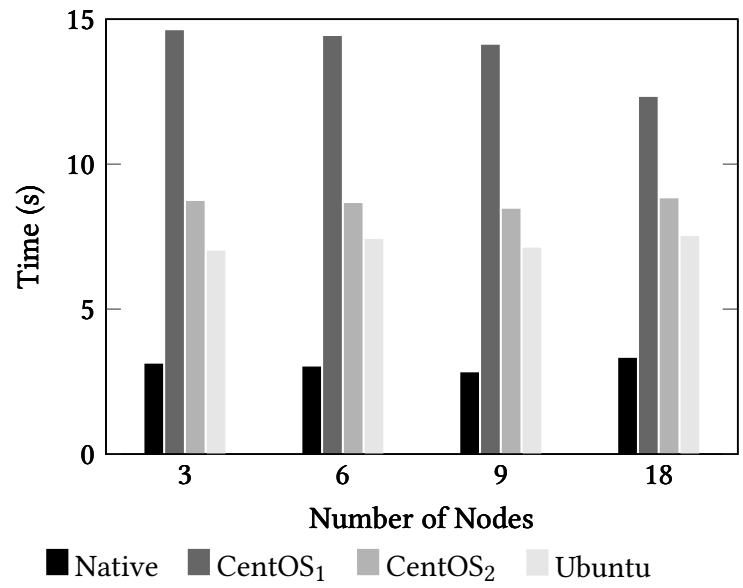

Figure 3: MILC startup time.

and the $\mathrm{CentOS}_{1}$ ones have the greatest among the three container environments.

Based on these benchmark results collected, we can calculate the container overhead from the difference in startup time of the container runs with the native ones. For the container overheads we calculated here, there is no dependency on the node counts, so we compute the average of all the container overheads for each application from across all node counts and use this as the measure of total container overhead for that test case (Figure ??).

The container overhead from $\mathrm{CentOS}_{1}$ is at least twice as large as the other two images (Figure ??). This is because of the excessive space taken by its built-in Intel Compiler stack, which makes the image size $19 \mathrm{~GB}$. The amount of time spent on loading the image dominates the overhead. We also looked at the performance characteristics [?] when running the WRF benchmarks trying to determine whether the large image size has impacts on the memory usage. No noticeable difference in memory usage is found compared with native runs. This is also in line with the fact that there is no 


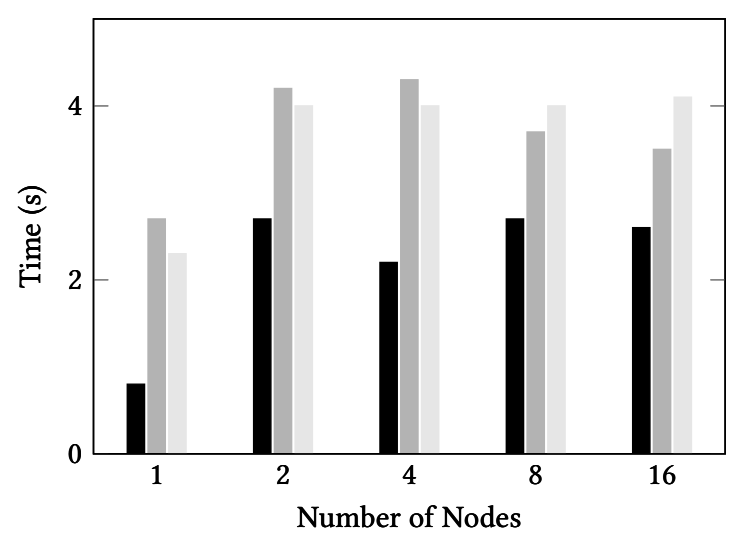

Native $\quad$ CentOS $_{2} \quad$ Ubuntu

Figure 4: NAMD startup time.

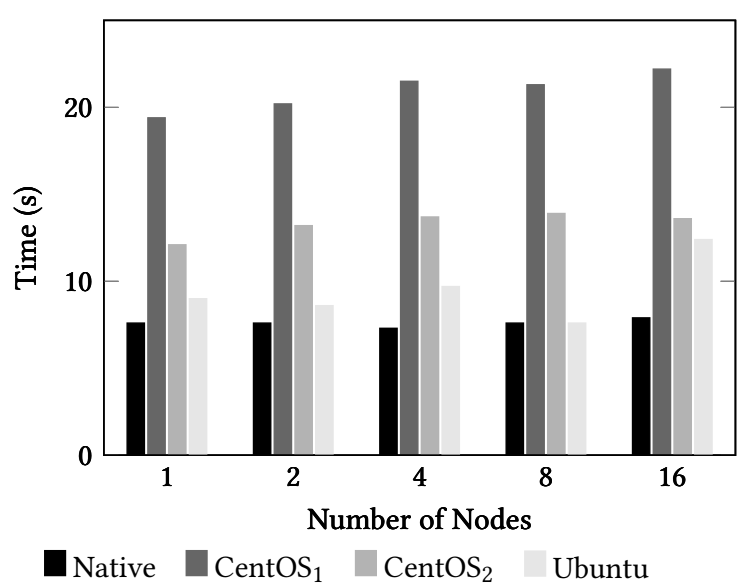

Figure 5: GROMACS startup time.

performance difference of $\mathrm{CentOS}_{1}$ shown in the runtime where overhead is neglected.

The overhead of Ubuntu is comparable to CentOS 2 's in WRF and NAMD, but slightly less in MILC and GROMACS (Figure ??). Considering the image sizes for $\mathrm{CentOS}_{2}$ and Ubuntu are $309 \mathrm{MB}$ and $212 \mathrm{MB}$ respectively, such a difference is not likely solely from loading the images. We suspect the difference in Linux kernel and system libraries between the two images also contributes to container's overhead. Such a small difference, however, is negligible given the comparable or even greater variance from noise and the orders of magnitude greater runtime for all the benchmark tests.

Overall, the container overhead stays constant with increasing node counts, so it would not deteriorate the scaling performance of HPC applications running within it. The overhead may vary among applications. However, as long as the container is lightweight in size, such overhead is harmless especially compared to the usually much longer runtime.

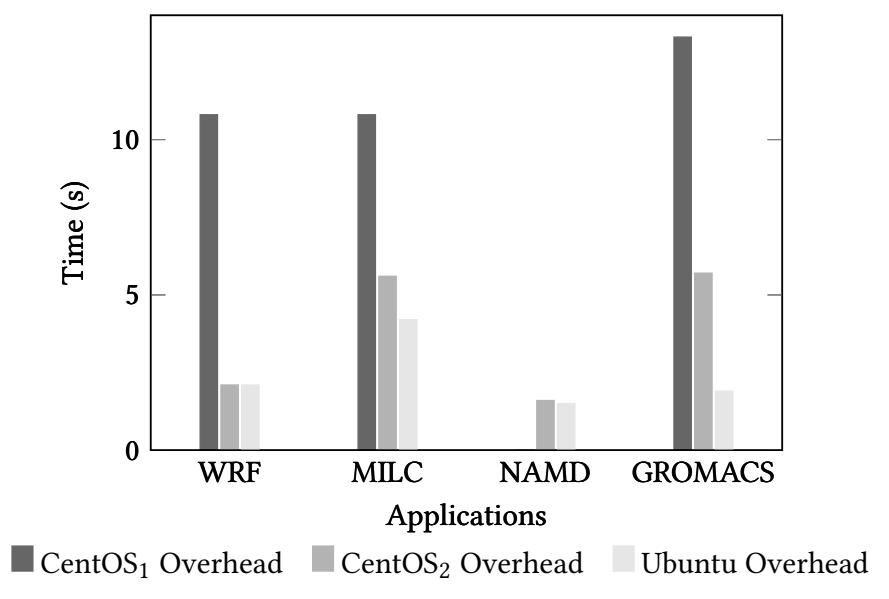

Figure 6: Average container overheads.

\section{CONCLUSION}

Our benchmark results indicate it is viable to provide a selection of customizable images to HPC users, and still have their applications achieve optimal performance when running within the Singularity container. Container images with excessive size may harm the performance by increasing the loading time, so lightweight containers are preferred. Only the basic tools and libraries as well as some minor patches were required to make the optimized applications compatible with the host HPC system. Here, we only tested two Linux distributions, CentOS and Ubuntu, that have a wide user base in the science community, but we expect similar images could also be implemented in the same way. Lightweight Singularity images can offer HPC users increased customizability, reproducibility and portability without sacrificing performance.

\section{ACKNOWLEDGMENTS}

This work is supported by the National Science Foundation through the Stampede2 (OAC-1540931) and XSEDE (ACI-1953575) awards. 